\title{
Critical considerations about the generalized use of $n-3$ polyunsaturated fatty acids in patients with Polycystic Ovary Syndrome
}

\author{
Maurizio Nordio ${ }^{1,2}$ \\ ${ }^{1}$ Department of Experimental Medicine, Sapienza University of Rome, Rome, Italy; ${ }^{2}$ The Experts Group on Inositol in Basic and Clinical Research \\ (EGOI), Rome, Italy \\ Correspondence to: Maurizio Nordio. Department of Experimental Medicine, Sapienza University of Rome, Rome, Italy. \\ Email: maurizionordio1@gmail.com. \\ Comment on: Yuan J, Wen X, Jia M. Efficacy of omega-3 polyunsaturated fatty acids on hormones, oxidative stress, and inflammatory parameters \\ among polycystic ovary syndrome: a systematic review and meta-analysis. Ann Palliat Med 2021;10:8991-9001.
}

Submitted Nov 20, 2021. Accepted for publication Jan 04, 2022.

doi: 10.21037/apm-21-3430

View this article at: https://dx.doi.org/10.21037/apm-21-3430

I've read with great interest the review recently published by Yuan et al. (1) about the efficacy of omega-3 polyunsaturated fatty acids (n-3 PUFAs) on hormonal, oxidative and inflammatory parameters in women with polycystic ovary syndrome (PCOS). The authors conducted a systematic review and a meta-analysis, finding that n-3 PUFA administration significantly improved (I) inflammatory and oxidative state, as indicated by the levels of C-Reactive Protein (CRP), Malondialdehyde (MDA) and Total Antioxidant Capacity (TAC), (II) and the hormonal profile, as reported by the levels of Luteinizing Hormone (LH), Total Testosterone (TT) and Sex Hormone Binding Protein (SHBG). However, the n-3 PUFA supplementation failed to improve other inflammatory, hormonal and oxidative factors in PCOS women, including Glutathione (GSH), Dehydroepiandrosterone Sulphate (DHEAS), Free Androgen Index (FAI) and Follicle-stimulating hormone (FSH). Indeed, the overall effects of n-3 PUFAs on the hormonal profile, oxidative stress, and inflammation in PCOS patients are still conflicting in the scientific literature.

Furthermore, the authors report in their discussion that some limitations to their work exist. First, the analysis included few eligible studies: in particular, among the 314 studies initially identified, only 10 met the eligible criteria and were included. Second, there is a wide heterogeneity among the identified studies, with increased risk of bias.

The authors conclude the analysis proposing the supplementation with n-3 PUFAs as a low-risk and addon therapy for patients with PCOS, without discussing crucial critical points. Based on current evidence, I strongly believe that the use of $n-3$ PUFAs in PCOS management needs deeper assessments regarding the effectiveness and the limitations concerning their application throughout women's life and their high cost.

EPA and DHA are two essential n-3 PUFAs, commonly used as dietary supplements for improving pathological cardiovascular parameters and inflammatory processes. Specifically, their use at different ratios and dosages correlates with different therapeutic benefits. The recommended minimum dosage for supplementation is $500 \mathrm{mg} /$ day, reaching $2,000-4,000 \mathrm{mg} /$ day in patients who have experienced myocardial infarction or altered blood triglycerides levels in the recent past. Therefore, considering such dosages and the high current cost associated to n-3 PUFAs (2), their use should be carefully assessed and reserved only to specific patients who may derive actual benefits from the treatment.

PCOS women exhibit heterogenous pathological features, besides the common diagnostic criteria of Rotterdam (oligo or amenorrhea, polycystic ovary, hyperandrogenism). Such syndrome indeed spans women's life, and menstrual and fertility problems evolve into metabolic complications as age advances (3). In particular, women after 40 years of age may commonly experience cardiovascular problems related to metabolic alterations 
including insulin resistance and obesity, dyslipidaemia, diabetes, hypertension. For this reason, the use of n-3 PUFAs should be limited to women over 40 years of age that have already experienced cardiovascular and inflammatory symptoms related to PCOS, while the generalized use in all types of PCOS patients seems unnecessary if not unadvisable.

Moreover, scientific evidence is still controversial about the beneficial effects of n-3 PUFAs on metabolic parameters. Some studies demonstrated that n-3 PUFA supplementation failed to improve symptoms related to PCOS, like body weight and hip circumference, fasting blood sugar, number of ovarian follicles, size of ovary, menstrual bleeding, and hirsutism score (4), as well as serum levels of total cholesterol (TC), LDL, HDL (4). On the contrary, other studies indicated positive effects only on TC and LDL, without affecting HDL and TG. In addition, a meta-analysis of randomized controlled clinical trials, conducted by Sadeghi and colleagues, revealed that supplementation with n-3 PUFAs may have not positive effects in improving insulin resistance in women with PCOS (4), corroborating the conflicting evidence.

On the other hand, the use of n-3 PUFAs deserves a proper attention in younger women with PCOS since guidelines recommend avoiding EPA administration during pregnancy (5). Indeed, EPA competes with arachidonic acid for the enzymes responsible for the formation of eicosanoids (cyclooxygenase, lipoxygenase), which are crucial for neural foetal development. In addition, like all other fatty acids, n-3 PUFAs exhibit a higher calorific value compared to other dietary supplements, and they should be taken with care in obese PCOS women.

Finally, I should point out that the use of n-3 PUFAs may have side effects that need to be monitored during the assumption, including gastrointestinal symptoms, like heartburn and nausea. Based on available information, supplementation with n-3 PUFAs in PCOS patients deserves a proper attention and it should be avoided in younger patients and in those with obesity, for which it seems unnecessary (6). On the contrary, widely recommended treatments for PCOS women are based on myo-inositol and D-chiro-inositol, which exhibit a lower calorific value than fatty acids, and well-established positive effects on hyperandrogenism and metabolic profile. Specifically, myo-inositol supplementation, by recovering the FSH signalling, counteracts the ovarian depletion, which determines menstrual irregularities and reduced fertility in PCOS women. Noteworthy, some studies conducted on different cellular systems reported that the stimulation with n-3 PUFAs may negatively modulate inositol trisphosphate pathway, also reducing its release as second messenger (7-9). A research group further hypothesized that in vitro stimulation with DHA may induce in cells of Saccharomyces Cerevisiae a depletion of intracellular inositol levels even stronger than valproic acid (10). Therefore, considering that women with PCOS exhibit a compromised inositol pathway, further studies are encouraged to make the clinical use of n-3 PUFAs in PCOS patients clearer.

In conclusion, we must always keep in mind that the management of PCOS aims to alleviate the symptoms and it may vary depending on patients' age and needs. On these bases, considering the nutritional characteristics and their cost, n-3 PUFA supplementation should not be generally recommended to all PCOS patients, but likely limited to those over 40 years with cardiometabolic disturbances.

\section{Acknowledgments}

Funding: None.

\section{Footnote}

Provenance and Peer Review: This article was a standard submission to the journal. The article did not undergo external peer review.

Conflicts of Interest: The author has completed the ICMJE uniform disclosure form (available at https://apm. amegroups.com/article/view/10.21037/apm-21-3430/coif) and has no conflicts of interest to declare.

Ethical Statement: The author is accountable for all aspects of the work in ensuring that questions related to the accuracy or integrity of any part of the work are appropriately investigated and resolved.

Open Access Statement: This is an Open Access article distributed in accordance with the Creative Commons Attribution-NonCommercial-NoDerivs 4.0 International License (CC BY-NC-ND 4.0), which permits the noncommercial replication and distribution of the article with the strict proviso that no changes or edits are made and the original work is properly cited (including links to both the formal publication through the relevant DOI and the license). 
See: https://creativecommons.org/licenses/by-nc-nd/4.0/.

\section{References}

1. Yuan J, Wen X, Jia M. Efficacy of omega-3 polyunsaturated fatty acids on hormones, oxidative stress, and inflammatory parameters among polycystic ovary syndrome: a systematic review and meta-analysis. Ann Palliat Med 2021;10:8991-9001.

2. Patterson AC, Stark KD. Direct determinations of the fatty acid composition of daily dietary intakes incorporating nutraceuticals and functional food strategies to increase $n-3$ highly unsaturated fatty acids. J Am Coll Nutr 2008;27:538-46.

3. Louwers YV, Laven JSE. Characteristics of polycystic ovary syndrome throughout life. Ther Adv Reprod Health 2020;14:2633494120911038.

4. Khani B, Mardanian F, Fesharaki SJ. Omega-3 supplementation effects on polycystic ovary syndrome symptoms and metabolic syndrome. J Res Med Sci 2017;22:64.

5. Helland IB, Smith L, Saarem K, et al. Maternal supplementation with very-long-chain n-3 fatty acids during pregnancy and lactation augments children's IQ at

Cite this article as: Nordio M. Critical considerations about the generalized use of $\mathrm{n}-3$ polyunsaturated fatty acids in patients with Polycystic Ovary Syndrome. Ann Palliat Med 2022;11(1):407409. doi: 10.21037/apm-21-3430
4 years of age. Pediatrics 2003;111:e39-44.

6. EMA Confirms Omega-3 Fatty Acid Medicines Are Not Effective in Preventing Further Heart Problems after a Heart Attack. Available online: https://www.ema.europa. eu/en/news/ema-confirms-omega-3-fatty-acid-medicinesare-not-effective-preventingfurther

7. Nair SS, Leitch J, Garg ML. N-3 polyunsaturated fatty acid supplementation alters inositol phosphate metabolism and protein kinase $\mathrm{C}$ activity in adult porcine cardiac myocytes. J Nutr Biochem 2001;12:7-13.

8. Sperling RI, Benincaso AI, Knoell CT, et al. Dietary omega-3 polyunsaturated fatty acids inhibit phosphoinositide formation and chemotaxis in neutrophils. J Clin Invest 1993;91:651-60.

9. Medini L, Colli S, Mosconi C, et al. Diets rich in n-9, n-6 and n-3 fatty acids differentially affect the generation of inositol phosphates and of thromboxane by stimulated platelets, in the rabbit. Biochem Pharmacol 1990;39:129-33.

10. Lee JY. The Effects of Omega-3 Fatty Acids on Intracellular Inositol Levels in Saccharomyces Cerevisiae. Available online: https://digitalcommons.andrews.edu/cgi/ viewcontent.cgi?article $=1139 \&$ context=honors 